\title{
Performance Evaluation of Energy Detector Based Spectrum Sensing for Cognitive Radio using NI USRP-2930
}

\author{
F. Z. El Bahi, H. Ghennioui, and M. Zouak \\ Laboratoire Signaux, Systèmes et Composants (LSSC), Faculté des Sciences et Techniques de Fès (FSTF) \\ Université Sidi Mohamed Ben Abdellah (USMBA), Route Immouzzer, B.P. 2202, Fès-Maroc
}

\begin{tabular}{l}
\hline \hline Article Info \\
\hline Article history: \\
Received: Feb 18, 2017 \\
Revised: May 28, 2017 \\
Accepted: Jun 12, 2017 \\
\hline Keyword: \\
Energy Detector \\
Spectrum Sensing \\
Cognitive Radio \\
Primary User \\
Secondary User \\
USRP \\
MATLAB
\end{tabular}

\begin{abstract}
This paper presents the performance evaluation of the Energy Detector technique, which is one of the most popular Spectrum Sensing (SS) technique for Cognitive Radio (CR). SS is the ability to detect the presence of a Primary User (PU) (i.e. licensed user) in order to allow a Secondary User (SU) (i.e unlicensed user) to access PU's frequency band using CR, so that the available frequency bands can be used efficiently. We used for implementation an Universal Software Radio Peripheral (USRP), which is the most used Software Defined Radio (SDR) device for research in wireless communications. Experimental measurements show that the Energy Detector can obtain good performances in low Signal to Noise Ratio (SNR) values. Furthermore, computer simulations using MATLAB are closer to those of USRP measurements.
\end{abstract}

Copyright (c) 2017 Institute of Advanced Engineering and Science. All rights reserved.

Corresponding Author:

Fatima Zahra EL BAHI,

Laboratoire Signaux, Systèmes et Composants (LSSC), Faculté des Sciences et Techniques de Fès (FSTF),

Université Sidi Mohamed Ben Abdellah (USMBA), Route Immouzzer, B.P. 2202, Fès-Maroc.

Email: fatimazahra.elbahi@usmba.ac.ma

\section{INTRODUCTION}

In recent years, the wireless applications and devices have developed and increased rapidly. Since the access to electromagnetic spectrum is fixed and limited, thus the available frequency bands are inefficiently utilized which causes the spectrum scarcity problem [1]. In order to use the available spectrum efficiently, many studies and researches have proposed a new concept called Cognitive Radio (CR). This concept is based on the opportunistic usage of radio frequency bands by allowing Secondary Users (SUs, i.e. unlicensed users) to exploit frequency bands of Primary Users (PUs, i.e. licensed users). CR is a radio for wireless communications able to change its parameters, related to either transmission or reception, autonomously and dynamically based on the electromagnetic environment and communication requirements, in order to perform an efficient communication without interfering with PUs [2].

CR has the capability of sensing the spectrum, thus detect the presence PU's signal, using different spectrum sensing techniques. These techniques are subdivided into two categories: cooperative and non-cooperative sensing techniques. The first category is based on sharing information, in other words, the detection of Primary User's signal is performed by combining results from multiple cognitive radios that works together [3]. The most important advantage of this technique is the capability of decreasing sensing time and improving the sensing accuracy. The second category, non-cooperative sensing techniques, is also known as primary transmitter detection, because the detection of PU's signal is based only on the received signal at a SU. The most common sensing techniques that belong to this category are: energy detector [4], matched filter detector and cyclostationary detector [5]. Energy detector is the most popular spectrum sensing technique, it can also be considered as the most spectrum sensing technique used in practice because of its low implementation complexity. The concept of Energy detector is based only on computing the total energy of the received signal, then comparing is to a specified threshold in order to decide the presence or absence of a PU's signal, thus no prior knowledge of the PU's signal is required, only the variance of the noise is needed.

In this paper, we present an implementation of the Energy Detector Based Spectrum Sensing, using an Universal Software Radio Peripheral (USRP), a Software Defined Radio (SDR) device, in order to evaluate the performance 
of the spectrum sensing technique. We used for the implementation an NI USRP-2930, which belongs to the USRP series of the National Instruments' brand. For the programming, we used LabVIEW and MATLAB for both transmission and reception. We implemented for transmission an OFDM (Orthogonal Frequency Division Multiplexing) Signal, which is used as a Primary User's signal. We chooses the OFDM modulation because it is the most used in wireless communication, due to its high bandwidth efficiency [6], such as WiFi [7], 3GPP/LTE [8] (3rd Generation Partnership Project/Long Term Evolution) for the downlink, DVBT [9] (Digital Video Broadcast Terrestrial) and WiMAX [10] (Worldwide Interoperability for Microwave Access). The performance evaluation of the Energy Detector is based on experimental measurements obtained using USRP's receiver, which included the Energy Detector algorithm.

The rest of this paper is organized as follows. In section 2, we introduce the Energy Detector based sensing after defining the problem formulation. Section 3 defines the implementation details using NI USRP-2930. Finally, in section 4 , the experimental results obtained from the implementation are provided in order to illustrate the performance of Energy Detector.

\section{ENERGY DETECTOR BASED SENSING}

\subsection{Problem Formulation}

In our system model, a SU senses the presence of a PU. In order to perform a good detection of a spectrum opportunity, two hypotheses, $H_{0}$ and $H_{1}$ are defined respectively for the absence and the presence of a PU signal. Hence, our hypothesis model for transmitter detection can be expressed as follows:

$$
\left\{\begin{array}{ll}
H_{0}: & s(l)=n(l) \\
H_{1}: & s(l)=x(l)+n(l)
\end{array},\right.
$$

where, $s(l)$ represents the received data, $x(l)$ is the transmitted signal by the primary user and $n(l)$ denotes the White Gaussian noise independent from the transmitted signal, with zero mean and variance $\sigma_{n}^{2}$. From the two hypotheses, two probabilities describe the performance of the spectrum sensing technique: the false-alarm probability $P_{f a}$, which is the probability of declaring wrongly $H_{1}$ and the detection probability $P_{d}$, which is the probability of declaring correctly $H_{1}$. The main purpose of all spectrum sensing techniques is to maximize the detection probability for a low false-alarm probability.

\subsection{Energy Detector}

Energy Detector, also known as radiometry, is the most popular and widely used spectrum sensing technique because of its low computational and implementation complexities. It is a simple sensing technique that does not need prior knowledge of the PU's signal, only the value of the White Gaussian Noise is needed. Urkowitz [4] was the first to investigate the detection of an unknown signal in a White noise channel using the energy detector based sensing. The PU signal is detected by comparing the total energy of the received signal, over a specified time duration, with a threshold. Thus, the test statistics of the energy detector is written as:

$$
T_{E D}=\frac{1}{L} \sum_{l=0}^{L}|s(l)|^{2},
$$

where, $L$ denotes the size of the observation sequence.

The presence of a PU's signal is thus detected if the energy is greater than the threshold. The decision is then expressed as follows:

$$
T_{E D} \stackrel{H_{1}}{\underset{H_{0}}{\gtrless}} \lambda,
$$

where, $\lambda$ denotes the threshold.

The Probability Density Function (PDF) of the test statistics $T_{E D}$ can be modeled as a Gaussian distribution [11] according to the two hypothesis as follows:

$$
\begin{cases}H_{0}: & T_{E D} \sim \mathcal{N}\left(\sigma_{n}^{2}, \frac{\sigma_{n}^{4}}{L}\right) \\ H_{1}: & T_{E D} \sim \mathcal{N}\left(\sigma_{x}^{2}+\sigma_{n}^{2}, \frac{\left(\sigma_{x}^{2}+\sigma_{n}^{2}\right)^{2}}{L}\right)\end{cases}
$$


where, $\sigma_{n}^{2}$ represents the variance of the White Gaussian noise and $\sigma_{x}^{2}$ denotes the variance of the transmitted PU's signal.

Based on the PDF of the test statistics, the detection probability $P_{d}$ and the false-alarm probability $P_{f a}$ can be expressed as:

$$
\begin{aligned}
P_{f a} & =\operatorname{Pr}\left(T_{E D}>\lambda \mid H_{0}\right)=Q\left(\left(\frac{\lambda}{\sigma_{n}^{2}}-1\right) \sqrt{L}\right) \\
P_{d} & =\operatorname{Pr}\left(T_{E D}>\lambda \mid H_{1}\right)=Q\left(\left(\frac{\lambda}{\sigma_{n}^{2}}-\rho-1\right) \sqrt{\frac{L}{2 \rho+1}}\right),
\end{aligned}
$$

where, $\rho=\frac{\sigma_{x}^{2}}{\sigma_{n}^{2}}$ denotes the Signal to Noise Ratio (SNR) and $Q($.$) is the Marcum Q-function defined as: Q(y)=$ $\frac{1}{\sqrt{2 \pi}} \int_{y}^{\infty} e^{-\frac{u^{2}}{2}} d u$. For a target false-alarm probability, the value of the threshold $\lambda$ can be calculated by inverting the relation described in Eq. 6 as follows:

$$
\lambda=\sigma_{n}^{2}\left(\frac{Q^{-1}\left(P_{f a}\right)}{\sqrt{L}}+1\right),
$$

where, $Q^{-1}($.$) is the inverse Marcum Q-function.$

\section{IMPLEMENTATION DETAILS}

In this section, we provide the practical implementation details of the Energy Detector based sensing. We used for both transmission and reception an NI USRP-2930 (Universal Software Radio Peripheral) and a Desktop Computer with LabVIEW 2014 and MATLAB R2013a to control the USRP using a Gigabit Ethernet Cable as shown in Figure 1.

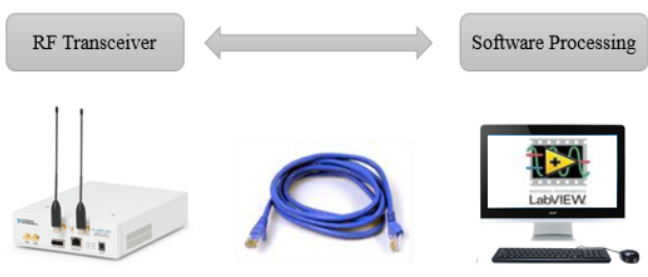

Figure 1. Implementation's structure

\subsection{NI USRP-2930}

NI USRP-2930 is a Software Defined Radio (SDR) transceiver, able to transmit and receive RF (Radio Frequency) signals, from the USRP series of the National Instruments' brand, it is widely used for both teaching and research in wireless communications. Furthermore, it enables a wide range of RF applications covering common standards such as GSM Cellular, broadcast radio, WiFi, GPS and digital TV. The USRP hardware is a straightforward $\mathrm{RF}$ platform for rapid prototyping applications such as spectrum monitoring and physical layer communication. It has the ability to transmit and receive RF signals across a frequency range from $50 \mathrm{MHz}$ to $2.2 \mathrm{GHz}$. Moreover, the NI USRP-2930 has an integrated GPS-disciplined clock that provides GPS position information, improved frequency accuracy and synchronization capabilities [12].

\subsection{Transmitter}

The programming and design parts of the transmitter are implemented in LabVIEW in order to control the NI USRP-2930. For our experiment, we consider an OFDM (Orthogonal Frequency Division Multiplexing) transmitted signal with a carrier frequency set to $200 \mathrm{Mhz}$.

As seen in Figure 2, the transmitter's front panel is divided into two parts. The left part contains three blocks; USRP Parameters, OFDM signal parameters and Debug (Figure 3). In the OFDM signal parameters block, we can choose the OFDM standard to transmit among the following ones: 3GPP/LTE, WiMax 802.16, DVBT-2K and 802.22-1K. The right part of the transmitter represents the PSD (Power Spectrum Density) of the OFDM transmitted signal. 


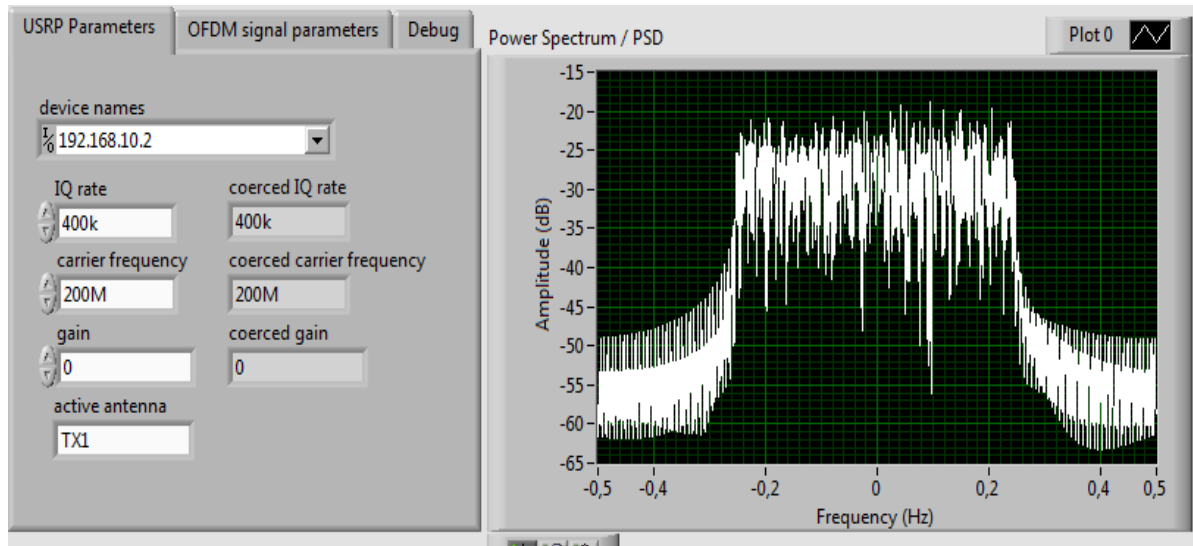

\section{田泪细}

Figure 2. Transmitter's front panel.

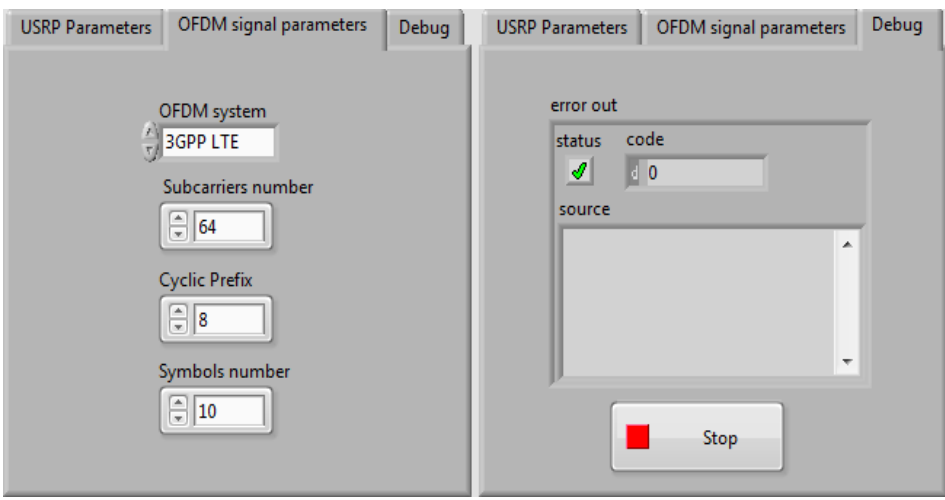

Figure 3. Transmitter's tab control.

\subsection{Receiver}

In the same way as the transmitter, we have implemented the receiver in LabVIEW as shown in Figure 4. The receiver's front panel is also divided into two parts, the left one contains three blocks; the USRP Parameters, Energy Detector and Debug. The receiver's debug part is the same as the one of the transmitter. In the Energy Detector part, as shown in Figure 5, we calculate the $P_{d}$ (Detection Probability) for a specific value of $P_{f a}$ (False-alarm Probability) and realizations. The right part of the receiver contains the PSD of the received OFDM signal, which is almost the same as the one of the OFDM transmitted signal.

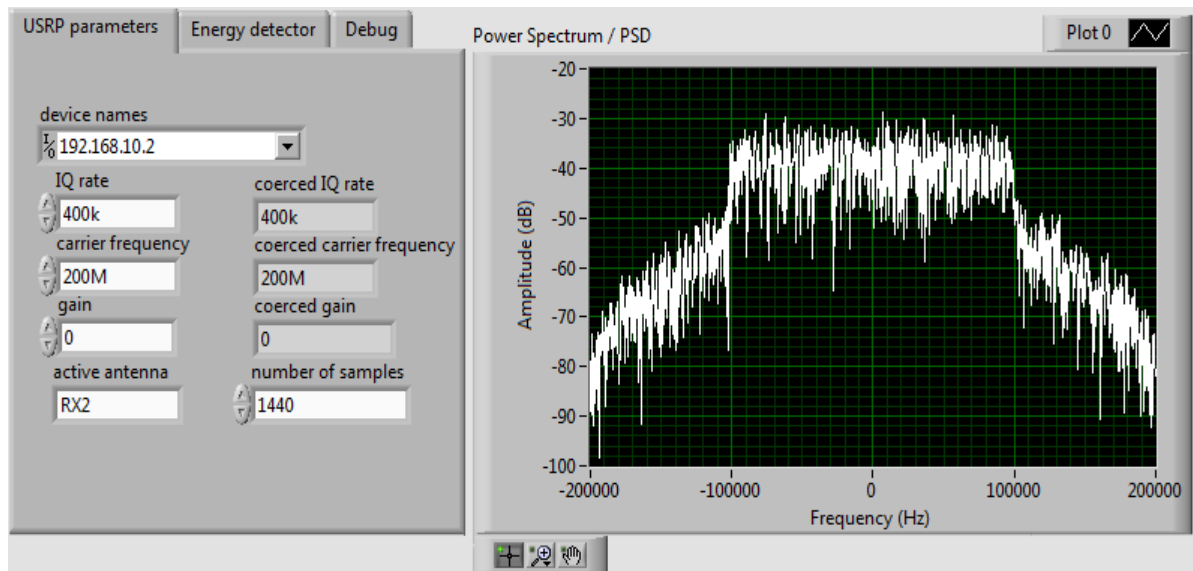

Figure 4. Receiver's front panel. 


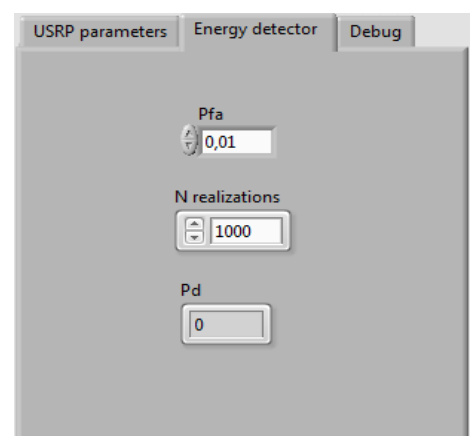

Figure 5. Receiver's tab control.

\section{RESULTS AND DISCUSSION}

In this section, we present experimental results of the implementation of the Energy Detector based sensing using NI USRP-2930 and averaged over 1000 realizations.

\subsection{Effect of SNR}

In this experiment, we test the impact of different SNR (Signal to Noise Ratio) values on the Detection Probability $\left(P_{d}\right)$. We fix the False-alarm Probability $\left(P_{f a}\right)$ to 0.01 and vary the SNR value from $-24 \mathrm{~dB}$ to $0 \mathrm{~dB}$ with a step of $2 \mathrm{~dB}$. Each measurement result is the average value of 1000 measurement results for the same SNR value. We generated an OFDM signal with 64 subcarriers, 10 symbols and cyclic prefix equals to 8 .

Figure $6 \mathrm{a}$ and $6 \mathrm{~b}$ show the MATLAB simulation (Computer simulations) and measurement results (USRP implementation) of the $P_{d}$ versus SNR of a 3GPP/LTE signal and DVBT-2K signal respectively. We can notice from both figures that the $P_{d}$ increases with the SNR values. Thus, the larger SNR, the better the detection of the OFDM PU. Furthermore, USRP implementations are closer to MATLAB simulations.

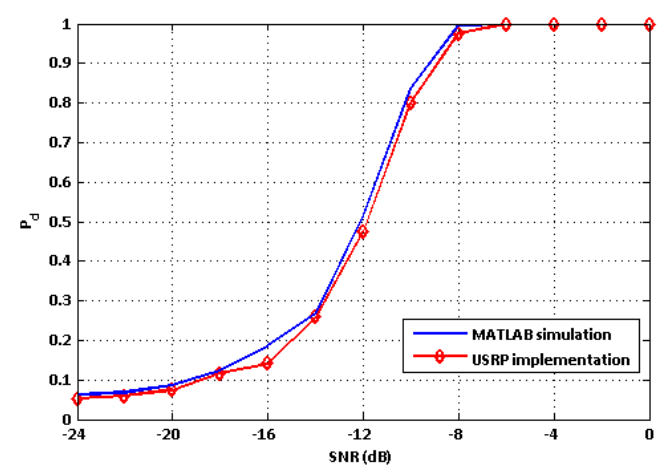

(a)

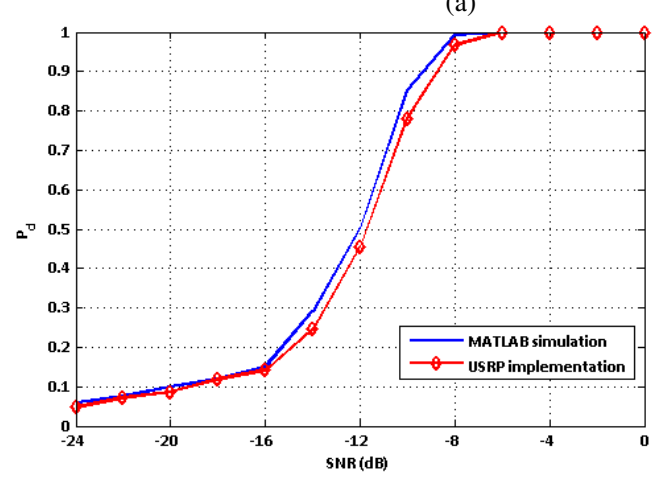

(b)

Figure 6. Detection probability $\left(P_{d}\right)$ vs. Signal to Noise Ratio (SNR) (with $P_{f a}=0.01$ ) of (a) 3GPP/LTE signal and (b) DVBT-2K signal. 


\subsection{Receiver Operation Characteristic}

In order to examine the effect of $P_{f a}$ on the detection performance of the Energy Detector, we fix the value of the SNR to $-10.4 \mathrm{~dB}$ and vary the $P_{f a}$ from 0.1 to 1 with a step of 0.1 . The achieved $P_{d}$ as a function of $P_{f a}$ is called the ROC (Receiver Operating Characteristic) curve. With the same way as the above experiment, each result is averaged over 1000 measurements.

Figure 7a and 7b represent the MATLAB simulation and USRP implementation of the $P_{d}$ versus $P_{f a}$ of a 3GPP/LTE signal and DVBT-2K signal respectively. As shown in those figures, USRP implementations are closer to MATLAB simulations and the Energy Detector achieves good performance for all $P_{f a}$ values.

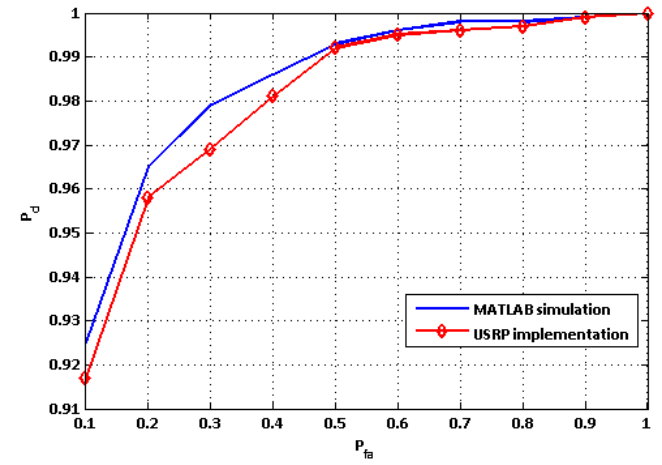

(a)

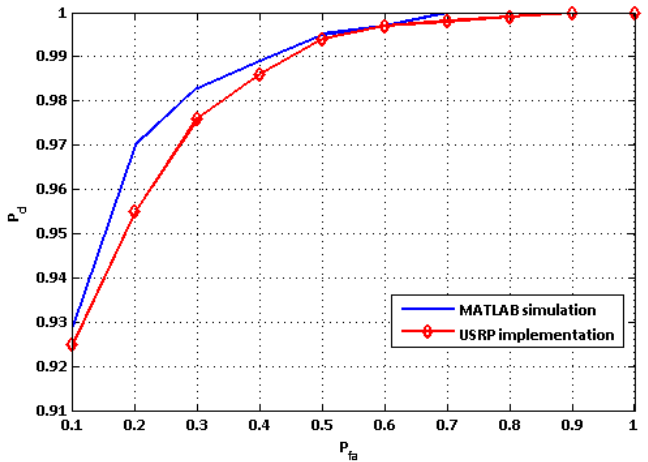

(b)

Figure 7. Detection probability $\left(P_{d}\right)$ vs. False-alarm probability $\left(P_{f a}\right)$ (with SNR=-10.4 dB) of (a) 3GPP/LTE signal and (b) DVBT-2K signal.

\section{CONCLUSION}

In this paper, we have provided an experimental performance evaluation of the Energy Detector based sensing using NI USRP-2930, which is a Software Defined Radio (SDR) transceiver. We have tested the impact of SNR on the detection probability. Furthermore, a ROC curve was obtained for a low SNR value. Experimental results shows that the Energy Detector achieves good performances for low SNR values and for all $P_{f a}$ values and are closer to those of computer simulations using MATLAB.

\section{REFERENCES}

[1] S. S. Ali, C. Liu, and M. Jin, "Minimum Eigenvalue Detection for Spectrum Sensing in Cognitive Radio," Int. J. Electr. Comput. Eng., vol. 4, no. 4, p. 623, 2014.

[2] Y. Saleem and M. H. Rehmani, "Primary radio user activity models for cognitive radio networks: A survey," $J$. Netw. Comput. Appl., vol. 43, pp. 116, 2014.

[3] M. S. Hossain, M. I. Abdullah, and M. A. Hossain, "Hard combination data fusion for cooperative spectrum sensing in cognitive radio," Int. J. Electr. Comput. Eng., vol. 2, no. 6, p. 811, 2012.

[4] H. Urkowitz, "Energy detection of unknown deterministic signals," Proc. IEEE, vol. 55, no. 4, pp. 523-531, 1967. 
[5] H. Sun, A. Nallanathan, C.-X. Wang, and Y. Chen, ”Wideband spectrum sensing for cognitive radio networks: a survey," IEEE Wirel. Commun., vol. 20, no. 2, pp. 7481, 2013.

[6] M. Hu, Y. Li, X. Lu, and H. Zhang, "Tone reservation to minimize nonlinearity impact on OFDM signals," IEEE Trans. Veh. Technol., vol. 64, no. 9, pp. 43104314, 2015.

[7] C. Smith and J. Meyer, "3G Wireless with WiMAX and WiFi: 802.16 and 802.11." McGraw-Hill Professional, 2005.

[8] H. Holma and A. Toskala, "HSDPA/HSUPA for UMTS: high speed radio access for mobile communications". John Wiley and Sons, 2007.

[9] U. Ladebusch and C. A. Liss, "Terrestrial DVB (DVB-T): A broadcast technology for stationary portable and mobile use," Proc. IEEE, vol. 94, no. 1, pp. 183193, 2006.

[10] O. A. Dobre, R. Venkatesan, and D. C. Popescu, "Second-order cyclostationarity of mobile WiMAX and LTE OFDM signals and application to spectrum awareness in cognitive radio systems," IEEE J. Sel. Top. Signal Process., vol. 6, no. 1, pp. 2642, 2012.

[11] Y.-C. Liang, Y. Zeng, E. C. Y. Peh, and A. T. Hoang, "Sensing-throughput tradeoff for cognitive radio networks," IEEE Trans. Wirel. Commun., vol. 7, no. 4, pp. 1326-1337, 2008.

[12] National Instruments Co., "USRP-292x/293x Datasheet," [Online]. Available: http://www.ni.com/datasheet/pdf/en/ds-355, accessed 3 February 2017.

\section{BIOGRAPHIES OF AUTHORS}

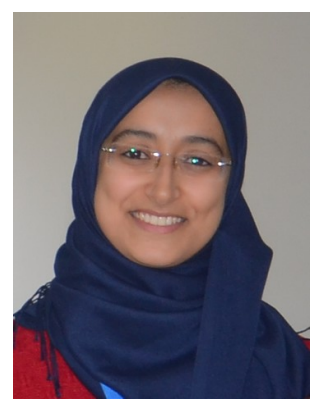

Fatima Zahra EL BAHI is an engineer in Network and Telecommunication, graduated in 2014 from National School of Applied Sciences of Tangier, Morocco. She is currently working toward $\mathrm{Ph} . \mathrm{D}$. degree in the laboratory of Signal, Systems and Components, in Faculty of Sciences and Technologies of Fez at Sidi Mohammed Ben Abdelah University, Morocco. Her main research interests are signal processing and cognitive radio.

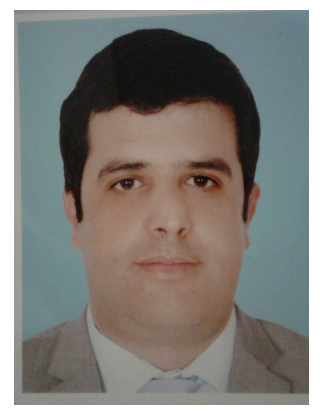

Hicham GHENNIOUI is an assistant director of Signals, Systems and Components Laboratory at Faculty of Sciences and Technologies, Fez, Morocco. Since 2011, he is a full-time Associate Professor at the Faculty of Sciences and Technologies, Fez. He received the Ph.D degree in Computer Science and Telecommunications in 2008, jointly from Mohamed V University and the Toulon University. In 2004, received the D.E.S.A. degree in Computer Science and Telecommunications from the Mohamed V University. His main research interests are signal/image processing including blind sources separation, data analytic, deblurring and cognitive radio.

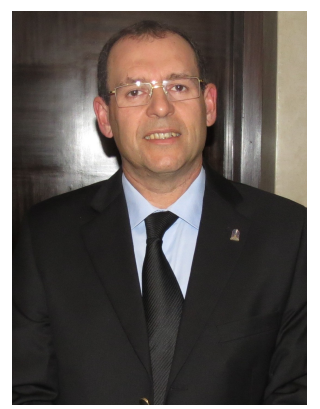

Mohcine ZOUAK is the director of the Cancer Research Institute of Fez, Morocco. President of the Conference of Deans of Faculties Scientific Morocco (CCMS). Vice President of International Conference of Heads of Universities and Scientific Institutions of French expression CIRUISEF. Since 2005, he is a professor of Higher Education at Faculty of Sciences and Technologies, Fez, Morocco. He assured lessons in the areas of signal processing, electronic systems and telecommunications as well as in the fields of stochastic estimation. His research activities mainly concern the signal processing and telecommunications. 